\title{
Vibration Characteristics Analysis of Composite Double-Layer Explosive Vessel Shell Subjected to Explosion Loading
}

\author{
Quan Wang $\left(\mathbb{D},{ }^{1,2}\right.$ Jie Gong, ${ }^{1}$ Zhimin Li, ${ }^{1}$ Shanghao Liu, ${ }^{1}$ \\ Chi-Min Shu, ${ }^{3}$ Yangfan Cheng, ${ }^{1}$ and Xuejiao $\mathrm{Li}^{1}$ \\ ${ }^{1}$ School of Chemical Engineering, Anhui University of Science and Technology, Huainan, Anhui 232001, China \\ ${ }^{2}$ Postdoctoral Research Station of Civil Engineering, Anhui University of Science and Technology, Huainan, Anhui 232001, China \\ ${ }^{3}$ Process Safety and Disaster Prevention Laboratory, National Yunlin University of Science and Technology, \\ Douliu, Yunlin 64002, Taiwan
}

Correspondence should be addressed to Quan Wang; wqaust@163.com

Received 21 October 2017; Revised 6 February 2018; Accepted 4 March 2018; Published 15 May 2018

Academic Editor: Sakdirat Kaewunruen

Copyright (C) 2018 Quan Wang et al. This is an open access article distributed under the Creative Commons Attribution License, which permits unrestricted use, distribution, and reproduction in any medium, provided the original work is properly cited.

\begin{abstract}
Explosive vessels are vulnerable to shock wave impacts during operation processing. It is necessary to explore the vessel's vibration characteristics. In this paper, acceleration sensors were adopted to collect vibration acceleration signals at the inner and outer surfaces of the composite double-layer explosive vessel under explosive loading. Then, the effective vibration velocity curve can be obtained by fitting polynomials to eliminate the acceleration integral trend. Thereafter, Hilbert-Huang Transform (HHT) was applied to analyze the time-frequency distribution of vibration velocity signals. The results showed that the special "steel plate-buffer interlayer-steel plate" structure can effectively attenuate the explosion vibration effect, and the frequency distribution range of vibration signal at the inner surface was wider than that at the outer surface, and furthermore the main vibration frequency was close to the natural frequency at the inner surface. Meanwhile, vibration amplitude and the main vibration frequency decreased obviously compared with the outer surface, and the vibration time of $0-200 \mathrm{~Hz}$ low-order frequency was shortened. The above researches closely linked the energy distribution with frequency of vibration signals and provided valuable reference for safer protection and vibration reduction design of explosive vessel.
\end{abstract}

\section{Introduction}

Explosive vessel is a type of special internal high-pressure antiknock container, which can effectively restrain the shock wave produced by explosions and limit the range of detonation products and fragments. Therefore, it can provide safer protection for personnel and the surrounding experimental instruments, which are essential experimental equipment for explosion testing $[1,2]$. The cylinder shell of explosive vessel may be subject to strong vibration and shock during application; the structural stability and antiknock performance are weakened accordingly. Thus, the researches on vibration characteristics of explosive vessel can provide theoretical basis for safer protection of explosive vessel. At present, the researches on explosive vessel are mainly focused on design, manufacture, and dynamic mechanical simulation analysis [3-7]. Kambouchev et al. [8, 9] computed the flow field corresponding to blast waves of different incident profiles propagating in air and impinging on free-standing plates by numerical method. Based on the achievements of Kambouchev's research, Vaziri and Hutchinson [10] studied the role of fluid-structure interaction in the design and assessment of sandwich plates subject to shocks. Dong et al. $[11,12]$ studied the cause of strain growth in explosive vessel based on the dynamic elastic buckling theory. However, the vibration spectrum analysis of the cylinder shell in the actual operation process is relatively scarce. Spectrum analysis can analyze the vibration signals of explosive vessel from two 
aspects of vibration action time and frequency distribution. To better evaluate the safety and guide the structural design for explosive vessel, it is necessary to delve into the spectrum analysis of vibration signals of explosive vessel.

In the early stage, the main methods for analyzing blasting vibration signals included Fourier transformation, fast Fourier transformation, short-time Fourier transformation, and wavelet packet transformation [13-15]. Fourier transformation is applicable to periodically stationary signals. Short-time Fourier transformation is unable to satisfy the requirements of frequency conversion [16, 17]. The window signal in wavelet packet analysis must be stationary and the choice of wavelet base function will have some influence on the analysis results $[18,19]$. While the blasting vibration signal is a typical nonstationary random signal, which has the characteristics of being complex, being irregular, short time, sudden change, and fast attenuation [20], the above analysis methods have some limitations.

Hilbert-Huang Transform (HHT) is a new analysis method for dealing with nonstationary random signals, which consist of empirical mode decomposition (EMD) and Hilbert-Huang spectral analysis, without predetermined basis functions and with the advantages of self-adaptability and high efficiency [16, 21]. Moreover, it has stronger local characteristics in signal processing, which can clearly characterize the energy specific distribution with the change of time and frequency. Therefore, it is widely used in all kinds of blasting signals analysis [22-27]. Li et al. [28] concluded that HHT is more adaptive than wavelet analysis in analyzing nonstationary signals. Zhang et al. [20] used HHT timefrequency analysis method to extract the frequency components of air explosion load signals in different periods. Zeng et al. [29] studied the spectral characteristics of the vibration signals measured on the building structure and the ground vibration signals by HHT.

The aim of this paper was to study vibration characteristics of composite double-layer structure explosive vessel during its actual operation processing. We collected and handled vibration signals produced on the inner and outer surfaces of the cylinder shell under explosive loading. According to the nonstationary and random characteristics of the blasting vibration signals, the time-frequency distribution characteristics of energy were analyzed by HHT method. The above analysis method and results may provide valuable reference for safer protection and protective design for some critical part of explosive vessel.

\section{Experimental Test on Explosive Vessel Cylinder Vibration}

2.1. Structural Dimensions of Explosive Vessel. The composite double-layer structure explosive vessel with ellipsoidal head was adopted in this paper, where the inner diameter is $3000 \mathrm{~mm}$, the inner steel plate thickness is $40 \mathrm{~mm}$, the outer steel plate thickness is $16 \mathrm{~mm}$, and buffer materials (foamed plastics) are filled between the inner and the outer walls. The cylinder is $2600 \mathrm{~mm}$ high with a volume of $24.943 \mathrm{~m}^{3}$, and both ends of the cylinder are standard 1:2 ellipsoidal heads. The explosive vessel is made of $16 \mathrm{MnR}$ steel, the elastic

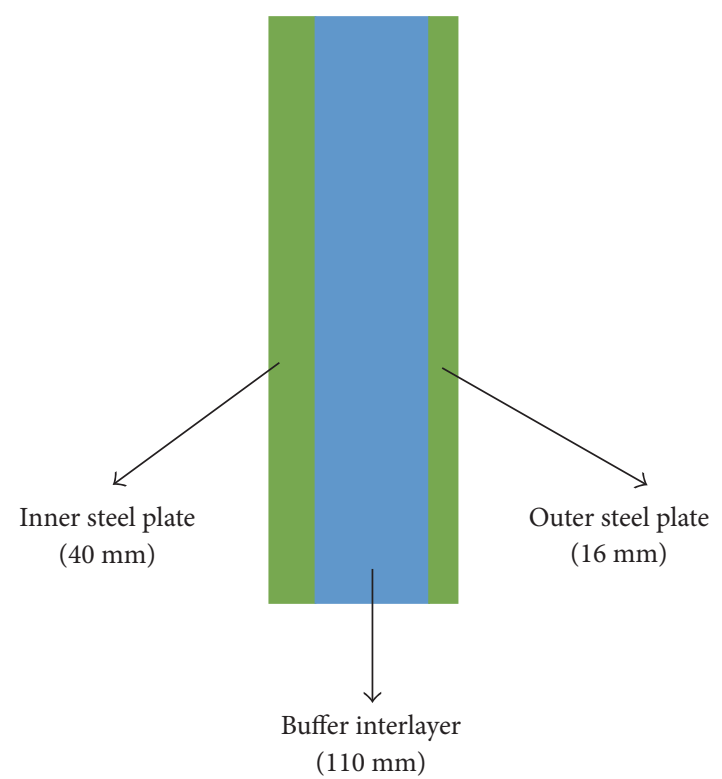

FIGURE 1: Schematic diagram for the special "steel plate-buffer interlayer-steel plate" structure.

modulus $E=208 \mathrm{GPa}$, Poisson's ratio $v=0.3$, and density $\rho=7.8 \times 10^{3} \mathrm{~kg} / \mathrm{m}^{3}$. The schematic diagram of the special "steel plate-buffer interlayer-steel plate" composite structure is shown in Figure 1.

The foam plastics have the characteristics of attenuating the stress amplitudes and prolonging the stress wave propagating time, which make it have effective impact resistance and buffering characteristics. The attenuation of the stress wave in the foam plastics is mainly caused by the constitutive properties of the foam core. The viscous effect of foam plastics attenuates and diffuses the amplitude of the stress wave during the propagation process with the propagation distance. The foam plastics porosity used in the paper is $85 \%-90 \%$, and its density is $30 \mathrm{~kg} / \mathrm{m}^{3}$.

2.2. Natural Vibration Frequency of Explosive Vessel. Angular frequency of cylindrical shell of explosive vessel can be calculated as follows [30]:

$$
\begin{aligned}
& \omega_{m n} \\
& =\sqrt{\frac{(m \pi R / L)^{4}}{\left[(m \pi R / L)^{2}+n^{2}\right]^{2}}+\frac{(\delta / R)^{2}}{12\left(1-v^{2}\right)}\left[\left(\frac{m \pi R}{L}\right)^{2}+n^{2}\right]^{2}} \sqrt{\frac{E}{\rho R^{2}}},
\end{aligned}
$$

where $m$ and $n$ are the axial and circumferential half wave number of the cylindrical shell, respectively. $L$ and $\delta$ are the length and thickness of the cylindrical shell, respectively.

Considering the restraint action of ellipsoidal heads, taking $m=1$, the natural vibration frequencies at inner and outer surfaces of explosion vessel shell are 456 and $421 \mathrm{~Hz}$, respectively.

2.3. Vibration Attenuation Effect Analysis of Buffer Interlayer. When the shock wave propagates in different materials, the shock wave impedance of each layer is different, and 
TABLE 1: Experimental setting parameters of oscilloscope.

\begin{tabular}{lcccc}
\hline Oscilloscope & $\begin{array}{c}\text { Vertical axis range } \\
(\mathrm{v} / \mathrm{div})\end{array}$ & Trigger level $(\mathrm{mv})$ & $\begin{array}{c}\text { Cross axle range } \\
(\mathrm{ms} / \mathrm{div})\end{array}$ & $\begin{array}{c}\text { Sampling frequency } \\
(\mathrm{kHz})\end{array}$ \\
\hline Parameters & 3.00 & 60 & 100 & 200 \\
\hline
\end{tabular}

transmission wave and reflection wave are produced at the interface of two layers of the material. According to the superposition principle and the condition of particle velocity and stress equal at the interface, when the wave impedance of the materials on both sides of the interface is different, the intensities of transmission wave (i) and reflection wave (ii) can be calculated as follows:

$$
\begin{aligned}
& \text { (i) } \sigma_{T 2}=T \sigma_{1} \text {, } \\
& \text { (ii) } \sigma_{R 1}=F \sigma_{1},
\end{aligned}
$$

where $\sigma_{T}$ is the transmission wave intensity, $\sigma_{R}$ is the reflection wave intensity, and subscripts 1 and 2 represent steel plate and foam cushioning material, respectively. $\sigma_{0}$ is the intensity of incident stress wave, $T$ is transmission coefficient (i), and $F$ is reflection coefficient (ii).

$$
\begin{aligned}
& \text { (i) } T=\frac{2 \rho_{2} c_{2}}{\rho_{1} c_{1}+\rho_{2} c_{2}}, \\
& \text { (ii) } F=\frac{\rho_{2} c_{2}-\rho_{1} c_{1}}{\rho_{1} c_{1}+\rho_{2} c_{2}},
\end{aligned}
$$

where $\rho_{1}$ and $\rho_{2}$ are the density of steel plate and foam cushioning material and $c_{1}$ and $c_{2}$ are the elastic wave velocity in steel plate and foam cushioning material. Among them, $\rho_{1}=7.8 \times 10^{3} \mathrm{~kg} / \mathrm{m}^{3}, c_{1}=5000 \mathrm{~m} / \mathrm{s}, \rho_{2}=30 \mathrm{~kg} / \mathrm{m}^{3}$, and $c_{2}=250 \mathrm{~m} / \mathrm{s}$.

The stress magnitude of transmitting into the buffer interlayer went through steel plate-buffer interlayer interface as $\sigma_{T 2}=T_{1} \sigma_{1}=0.000385 \sigma_{1}$. While the stress wave passes through the buffer layer medium into the rear steel plate, the stress magnitude in the rear steel plate medium is $\sigma_{\mathrm{T} 3}=$ $T_{2} \sigma_{T 2}=0.00711 \sigma_{1}$.

The theoretical calculation shows that, due to the existence of foam buffer interlayer, the stress wave passes through the special "steel plate-buffer interlayer-steel plate" structure, and the peak value of stress is reduced to 7.14 percent. This indicates that the buffer interlayer can effectively attenuate the stress peak value acting on the steel plate so as to decrease the damage caused by the explosion shock wave.

2.4. Experimental Test System. The middle section of the cylinder shell is nearest to the explosion source, which bears the largest explosive loading. Therefore, the vibration signals at the middle section of explosion source are tested in this experiment. The test system mainly consists of acceleration sensor (type: CA-YD-121, sensitivity: $200 \mathrm{mv} / \mathrm{g}$ ), constant current source, and oscilloscope (type: Lecroy HDO4030). The spherical emulsion explosive charge with mass of $50 \mathrm{~g}$ was located in the center of the explosive container, which was at a $1.5 \mathrm{~m}$ horizontal distance from the inner wall and $1.6 \mathrm{~m}$ height from the bottom of the explosive container. Acceleration sensors $a$ and $b$ were, respectively, arranged at the symmetrical position of the inner and outer surfaces of the explosive vessel, which had the same height as the explosive charge. Then, acceleration vibration signals at the inner and outer surfaces of the cylinder shell were measured by acceleration sensors $a$ and $b$. The setting parameters of the oscilloscope are listed in Table 1, and the schematic diagram of the test system is shown in Figure 2.

\section{Experimental Test Results}

3.1. Vibration Acceleration Signals. The vibration acceleration signals at inner and outer surfaces of the explosive vessel are monitored by acceleration sensors $a$ and $b$, as shown in Figures 3 and 4 .

As can be seen from Figures 3 and 4, the vibration acceleration at inner and outer surfaces of the cylinder shell rose to a maximum value after explosive detonation of 0.3 and $0.2 \mathrm{~ms}$, respectively. Furthermore, the peak value of vibration acceleration at the inner surface was obviously higher than that of the outer surface, which is mainly attributed to composite structure of the explosive vessel; that is, there existed buffer material between the inner wall and the outer wall.

3.2. Acceleration Integral Trend Item. Assuming that the acceleration signal is $a_{0}(t)$, which is integrated to obtain a velocity signal,

$$
v(t)=\int_{0}^{t} a_{0}(t) d t=v^{\prime}(t)+v_{0}
$$

where $v_{0}$ is the initial speed and $v^{\prime}(t)$ is the primitive function of $a_{0}(t)$.

The actual collected vibration acceleration signal $a(t)$ contains DC components $\varepsilon$; that is, $a(t)=a^{\prime}(t)+\varepsilon$. The integration of minor DC components generates integral trend item.

$$
\begin{aligned}
v(t) & =\int_{0}^{t} a(t) d t=\int_{0}^{t}\left[a_{0}(t)+\varepsilon\right] d t \\
& =v^{\prime}(t)+v_{0}+\varepsilon t+\eta .
\end{aligned}
$$

3.3. Elimination of Integral Trend Item by Fitting Polynomial. The obtained velocity signals by integrating directly the acceleration signals contain the integral trend term. To eliminate the integral trend item, the acceleration signals can be processed by fitting a polynomial to obtain an effective time-velocity curve $[31,32]$, as illustrated in Figures 5 and 6. 


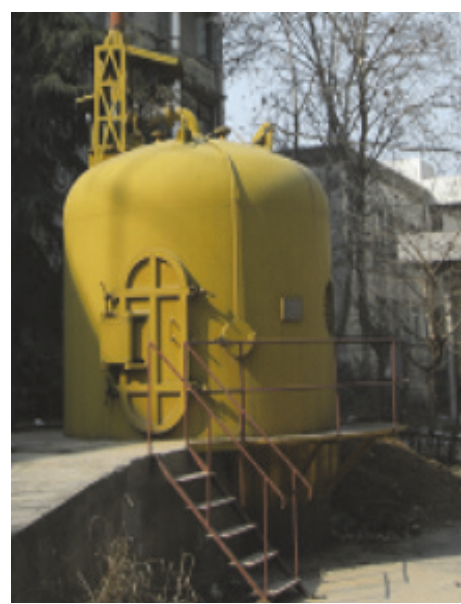

(a) Appearance of the explosive vessel

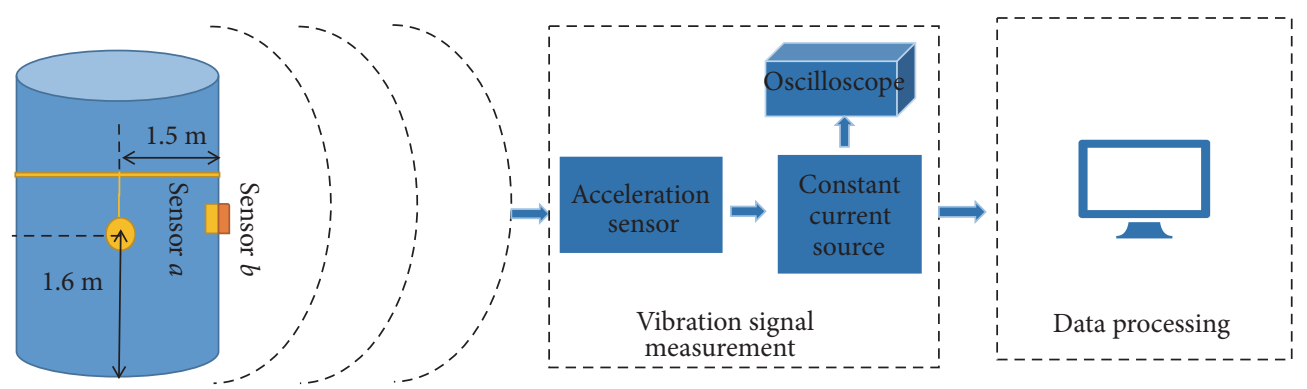

(b) Schematic diagram for testing

Figure 2: Experiment system.

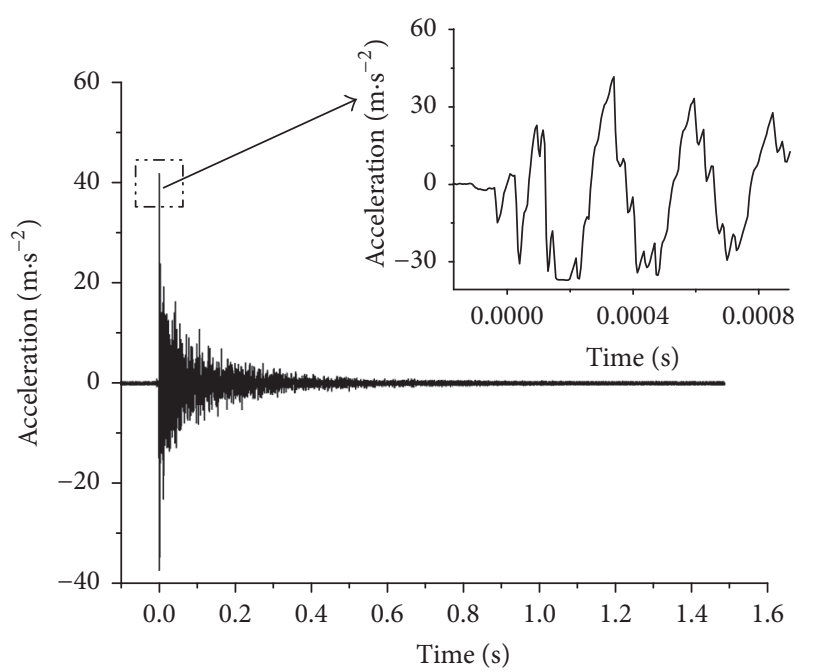

FIGURE 3: Vibration acceleration signal at inner surface of explosive vessel.

We can see clearly the time-velocity curves obtained by integrating the acceleration signals directly with the integral trend term, which cannot authentically reflect the change regulation of vibration velocity, while the efficient timevelocity curves were obtained by fitting the polynomial, which provide valid waveform data for further analyzing the energy distribution of vibration signals.

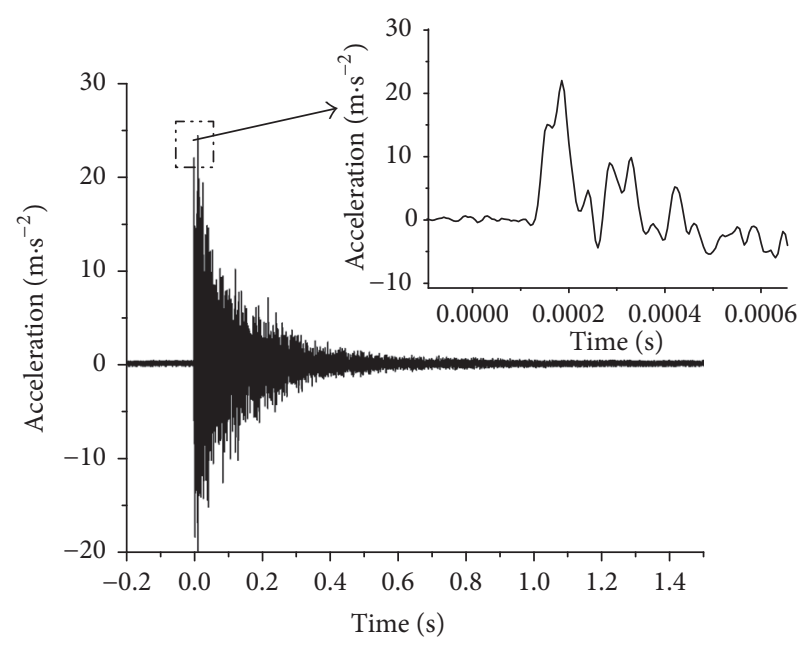

FIGURE 4: Vibration acceleration signal at outer surface of explosive vessel.

\section{HHT Analysis of Vibration Velocity Signals}

4.1. EMD for Vibration Velocity Signals. In order to find out all extreme points of the above vibration velocity signal $v(t)$ firstly, the upper and lower envelopes can be constructed, which cover all local maxima and local minima, respectively. Thereafter, the mean value $m_{1}(t)$ can be obtained by linking 


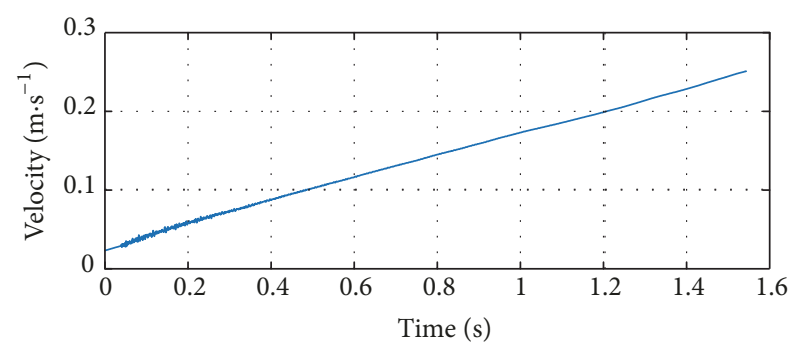

(a) Velocity curve obtained from integrating original acceleration signal

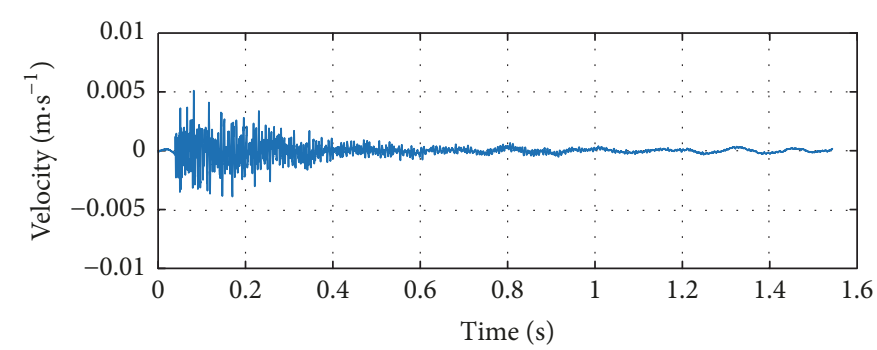

(b) Velocity curve obtained from elimination of integral trend item

FIGURE 5: Vibration velocity curve at inner surface of explosive vessel.

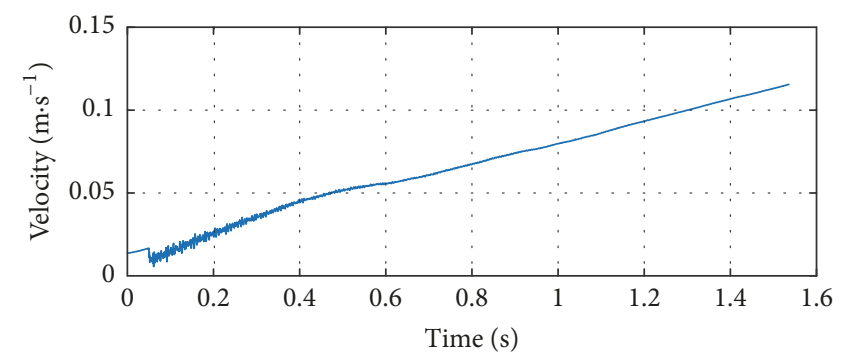

(a) Velocity curve obtained from integrating original acceleration signal

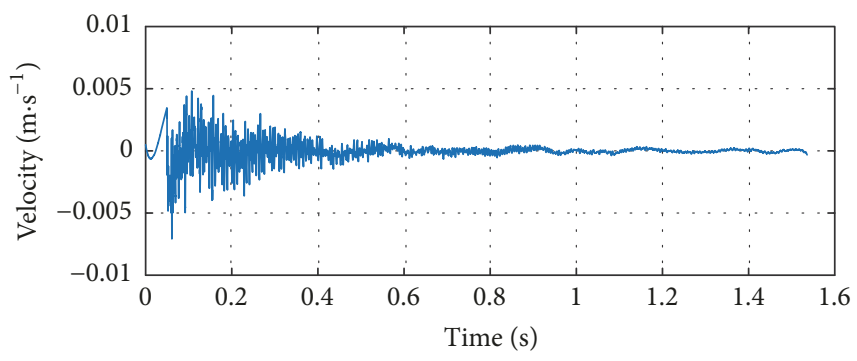

(b) Velocity curve obtained from elimination of integral trend item

FIGURE 6: Vibration velocity curve at outer surface of explosive vessel.

in sequence the upper and lower envelopes. The difference between $m_{1}(t)$ and $v(t)$ is denoted as $h_{1}(t)$ :

$$
h_{1}(t)=v(t)-m_{1}(t) .
$$

Determining whether $h_{1}(t)$ satisfies the two conditions of IMF function [17, 28]: (1) the number of extreme points and the number of zero crossings points must either be equal or differ at most by one in whole datasets; (2) the mean value of the envelope defined by the local maxima and the envelope defined by the local minima is zero at every point in the signal. IMFs are viewed as generalized simple harmonic functions that have time-varying amplitudes and frequencies.

If $h_{1}(t)$ satisfies the conditions, it is set to the first IMF component, that is, $\mathrm{imf}_{1}$. If the condition is not satisfied, $h_{1}(t)$ is set as the new original signal and subjected to the same sifting process until the qualified $h_{k}$ is sifted, which is recorded as the first IMF component. Now, the margin $r_{1}(t)$ could be calculated as follows:

$$
r_{1}(t)=v(t)-\mathrm{imf}_{1} .
$$

Then, $r_{1}(t)$ is taken as a new signal and the above procedure is repeated until the condition is satisfied with

$$
R=\sum_{i=0}^{k}\left|\frac{\left|v_{k}(t)-v_{k-1}(t)\right|^{2}}{v_{k}(t)}\right| \quad(R: 0.2-0.3) .
$$

The IMF components of the vibration velocity signals at the inner and outer surface of the explosive vessel were obtained by Matlab software programming, as shown in Figures 7 and 8.

Variance contribution rate is the percentage of each IMF component's variance and the sum of all IMF components' variance, which reflects the contribution magnitude in which each IMF component accounts for the motion energy of the whole sequence signal. In view of the aforementioned IMF components, the corresponding variance contribution rates are calculated, as presented in Tables 2 and 3 . The comparison result is shown in Figure 9.

The vibration signals are decomposed into a series of IMF components; here are some conclusions drawn from Figures 7 and 8.

The vibration velocity signal at the inner surface was decomposed into 18 IMF components and a margin $\operatorname{imf}_{19}$ as shown in Figure 7 . The frequency gradually decreased with the processing of EMD, and the amplitude of the components was greater, which indicates that the energy of this frequency band is stronger [33]. $\mathrm{imf}_{1}-\mathrm{imf}_{2}$ components had the highest frequency, but they accounted for very small percentage of energy and the variance contribution rates were low, which were related to the high-frequency noise in the experimental test. The energy amplitudes of $\mathrm{imf}_{3}-\mathrm{imf}_{6}$ components were larger, while the variance contribution rates were smaller. The amplitudes of $\mathrm{imf}_{7}-\mathrm{imf}_{14}$ components were also larger and the variance contribution rates of the IMF components were higher, which were the dominant frequency band of the signal. $\mathrm{imf}_{15}-\mathrm{imf}_{18}$ were the smaller frequency components after decomposition, which were weak vibration signals 


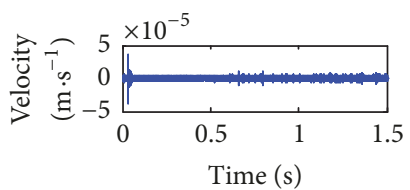

(a) $\operatorname{imf}_{1}$

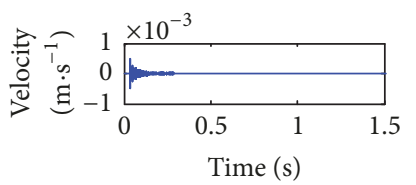

(e) $\mathrm{imf}_{5}$

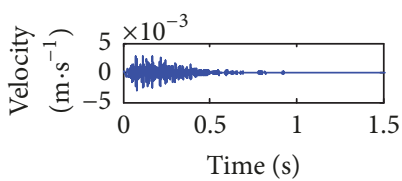

(i) $\operatorname{imf}_{9}$

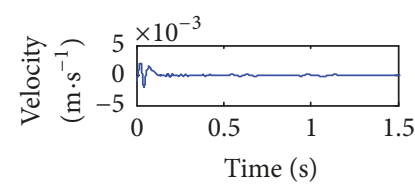

(m) $\operatorname{imf}_{13}$

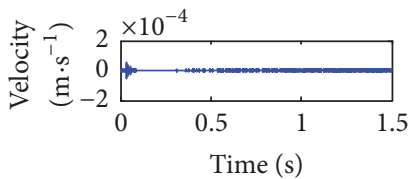

(b) $\mathrm{imf}_{2}$

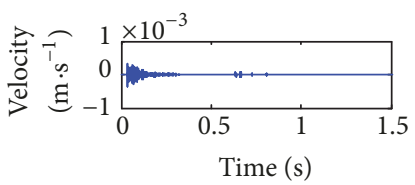

(f) $\operatorname{imf}_{6}$

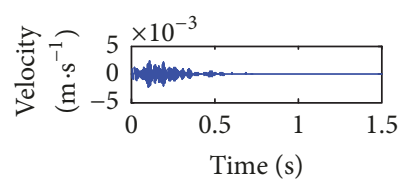

(j) $\operatorname{imf}_{10}$

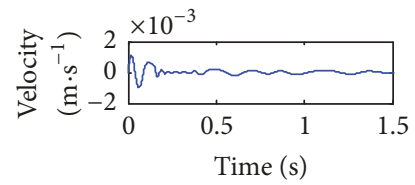

(n) $\operatorname{imf}_{14}$

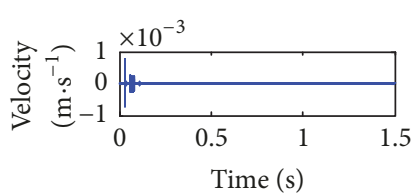

(c) $\operatorname{imf}_{3}$

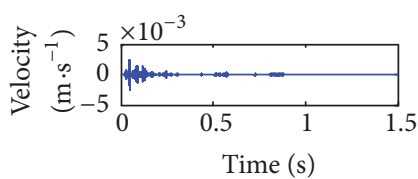

(g) $\operatorname{imf}_{7}$

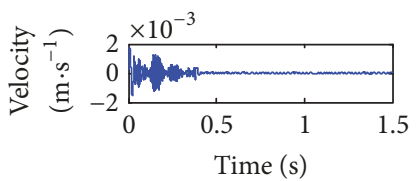

(k) $\operatorname{imf}_{11}$

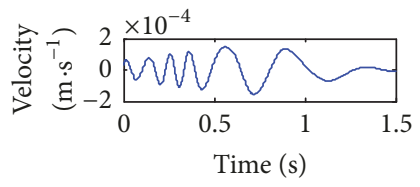

(o) $\operatorname{imf}_{15}$

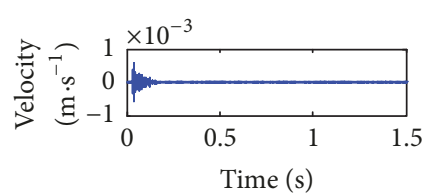

(d) $\operatorname{imf}_{4}$

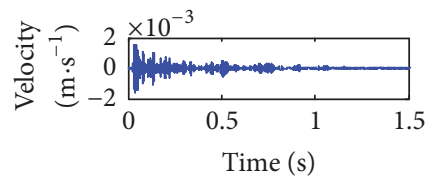

(h) $\mathrm{imf}_{8}$

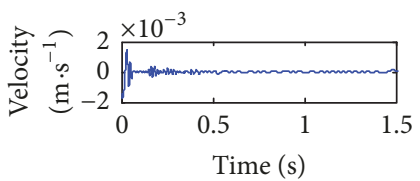

(l) $\operatorname{imf}_{12}$

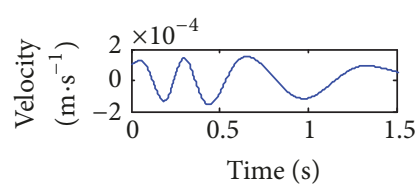

(p) $\operatorname{imf}_{16}$

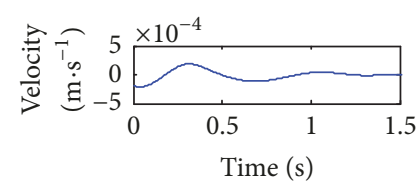

(q) $\operatorname{imf}_{17}$

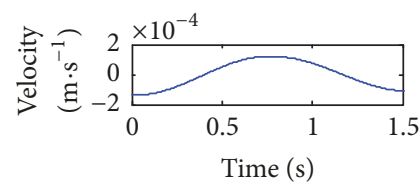

(r) $\operatorname{imf}_{18}$

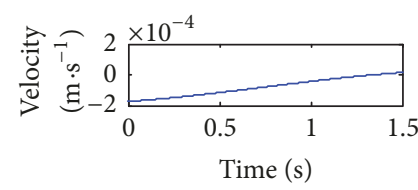

(s) $\operatorname{imf}_{19}$

FIGURE 7: IMF components of the vibration velocity at the inner surface of explosive vessel.

caused by explosion vibration acting on the contact surface between explosive vessel shell and the sensor. $\mathrm{imf}_{19}$ was the decomposition margin, which could be the weak trend term or the zero drift of the instrument.

The vibration velocity signal at the outer surface was decomposed into $16 \mathrm{IMF}$ components and a margin $\mathrm{imf}_{17}$ as shown in Figure 8. Among them, $\mathrm{imf}_{1}-\mathrm{imf}_{2}$ components had the highest frequency, but the percentage of energy and variance contribution rates of the IMF components were very low. $\mathrm{imf}_{3}-\mathrm{imf}_{6}$ components contained the high-frequency components; however, the variance contribution rates were low. The amplitudes of the $\mathrm{imf}_{7}-\mathrm{imf}_{12}$ components were the largest, and the contribution rates were higher, which were the dominant frequency section of the signal. $\mathrm{imf}_{13}-\mathrm{imf}_{16}$ were the low-frequency components of the signal, and $\mathrm{imf}_{17}$ was the decomposition margin.

4.2. Hilbert-Huang Spectrum of Vibration Velocity Signal. The instantaneous spectrum of the above IMF components can be obtained by Hilbert-Huang transformation; then, the
Hilbert-Huang spectrum can be obtained by synthesizing the instantaneous spectrum of all the IMF components:

$$
H[\operatorname{imf}(t)]=\frac{1}{\pi} \mathrm{PV} \int_{-\infty}^{\infty} \frac{\operatorname{imf}\left(t^{\prime}\right)}{t-t^{\prime}} d t^{\prime},
$$

where PV represents the Cauchy principal value. The HilbertHuang spectrum of vibration velocity signals is delineated in Figures 10 and 11, respectively.

Here are the following conclusions through HHT.

(1) The frequency distribution range of vibration velocity signal at the inner surface of explosive vessel was dispersed, low-frequency and high-frequency components all appeared, and energy fluctuation existed in the range of $0-5000 \mathrm{~Hz}$. The darker spots with large vibration energy were mainly distributed before $0.40 \mathrm{~s}$, and the corresponding maximum frequency was $1000 \mathrm{~Hz}$ in $0-0.10 \mathrm{~s}$. While the corresponding maximum frequency was $500 \mathrm{~Hz}$ in $0.10-0.40 \mathrm{~s}$, this frequency was close to the natural frequency at inner surface of explosive vessel, and explosive vessel may be damaged due to the resonance phenomenon and safer protection was considered seriously. 


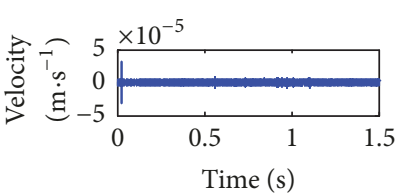

(a) $\mathrm{imf}_{1}$

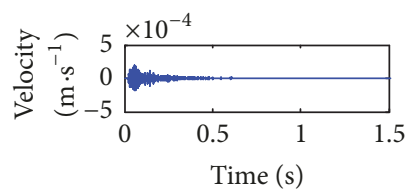

(e) $\operatorname{imf}_{5}$

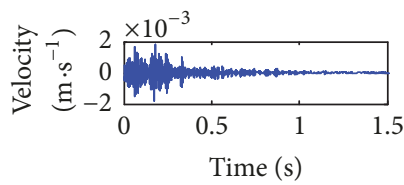

(i) $\mathrm{imf}_{9}$

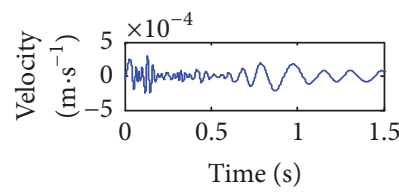

(m) $\mathrm{imf}_{13}$

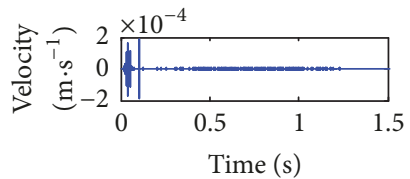

(b) $\operatorname{imf}_{2}$

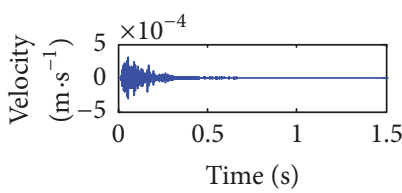

(f) $\operatorname{imf}_{6}$

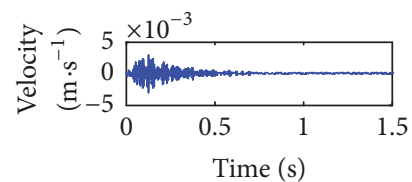

(j) $\operatorname{imf}_{10}$

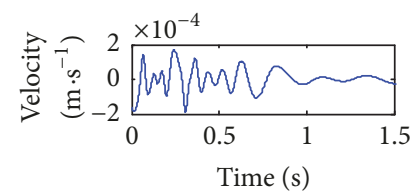

(n) $\operatorname{imf}_{14}$

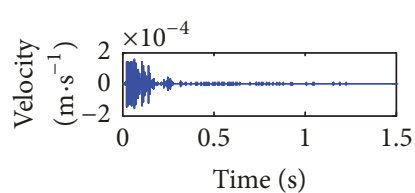

(c) $\mathrm{imf}_{3}$

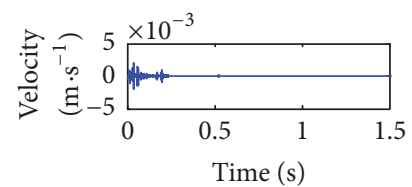

(g) $\operatorname{imf}_{7}$

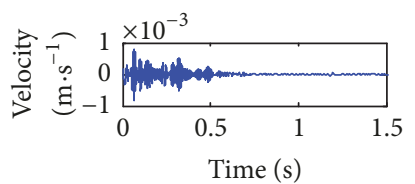

(k) $\operatorname{imf}_{11}$

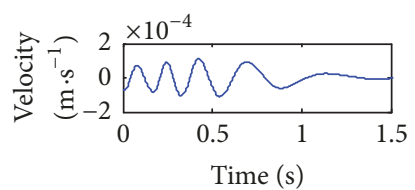

(o) $\operatorname{imf}_{15}$

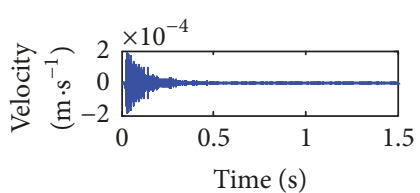

(d) $\mathrm{imf}_{4}$

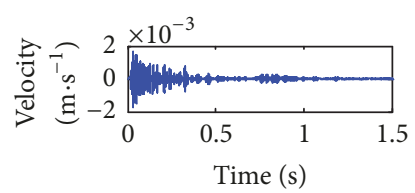

(h) $\operatorname{imf}_{8}$

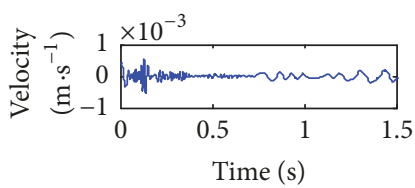

(1) $\operatorname{imf}_{12}$

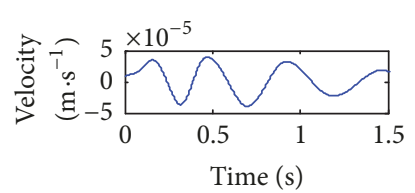

(p) $\operatorname{imf}_{16}$

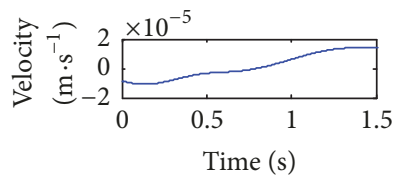

(q) $\operatorname{imf}_{17}$

FIGURE 8: IMF components of the vibration velocity at the outer surface of explosive vessel.

(2) The frequency distribution range of vibration velocity signal at outer surface of explosive vessel was relatively concentrated, mainly distributed in $0-1000 \mathrm{~Hz}$. The darker spots with large vibration energy were mainly distributed before $0.20 \mathrm{~s}$, the maximum frequency corresponding to $200 \mathrm{~Hz}$, away from the natural frequency at outer surface of explosive vessel. Therefore, the damage of explosive vessel caused by resonance phenomenon can be avoided.

\section{Discussion and Conclusions}

The HHT analysis method can effectively obtain the IMF components with definite physical meaning; meanwhile, the energy distribution and change regulation of vibration signals were analyzed from two aspects of vibration action time and frequency distribution, so as to better evaluate the safety of explosive vessel.

As can be seen from the HHT analysis of the vibration velocity signals that the frequency distribution of vibration velocity at inner surface of explosive vessel was wider, the response components with higher frequency were mainly concentrated in the initial stage of explosion and decreased gradually over time. The frequency distribution of the vibration velocity signal at outer surface of explosive vessel was more concentrated, which was mainly distributed in the lower frequency section of $0-200 \mathrm{~Hz}$. Because the outer surface of explosion vessel was not directly impacted by the explosion, the special "steel plate-buffer interlayer-steel plate" structure had certain protective effect, and the buffer interlayer effectively absorbs the energy produced by explosion. Consequently, the frequency distribution range was more concentrated, the vibration frequency was evidently smaller, and the action time of the low-order frequency section was obviously shortened.

According to the above discussion, concluding remarks could be made as follows.

(1) The vibration acceleration signals of the explosion vessel were analyzed by HHT, which is an efficient and accurate explosive signal processing method. Under the impact of explosion loading, the frequency range of vibration signal at inner surface was wider than that of outer surface. The main vibration frequency of the inner surface was close to its natural vibration frequency, which was the vulnerable part in the process of its application. 
TABLE 2: IMF components' variance contribution rates of vibration velocity signal at inner surface.

\begin{tabular}{lc}
\hline Component & Contribution rate/\% \\
\hline $\operatorname{imf}_{1}$ & 0.0011 \\
$\mathrm{imf}_{2}$ & 0.0008 \\
$\mathrm{imf}_{3}$ & 0.0305 \\
$\mathrm{imf}_{4}$ & 0.0530 \\
$\mathrm{imf}_{5}$ & 0.0524 \\
$\operatorname{imf}_{6}$ & 0.0697 \\
$\operatorname{imf}_{7}$ & 3.5825 \\
$\operatorname{imf}_{8}$ & 4.4773 \\
$\operatorname{imf}_{9}$ & 29.2185 \\
$\operatorname{imf}_{10}$ & 19.5410 \\
$\operatorname{imf}_{11}$ & 9.9252 \\
$\operatorname{imf}_{12}$ & 6.4797 \\
$\operatorname{imf}_{13}$ & 14.8017 \\
$\operatorname{imf}_{14}$ & 7.4380 \\
$\operatorname{imf}_{15}$ & 0.8120 \\
$\operatorname{imf}_{16}$ & 1.2113 \\
$\operatorname{imf}_{17}$ & 1.1991 \\
$\operatorname{Imf}_{18}$ & 1.1062 \\
\hline
\end{tabular}

TABLE 3: IMF components' variance contribution rates of vibration velocity signal at outer surface.

\begin{tabular}{lc}
\hline Component & Contribution rate/\% \\
\hline $\operatorname{imf}_{1}$ & 0.0023 \\
$\operatorname{imf}_{2}$ & 0.0052 \\
$\operatorname{imf}_{3}$ & 0.0306 \\
$\operatorname{imf}_{4}$ & 0.0497 \\
$\operatorname{imf}_{5}$ & 0.0698 \\
$\operatorname{imf}_{6}$ & 0.1655 \\
$\operatorname{imf}_{7}$ & 5.5737 \\
$\operatorname{imf}_{8}$ & 10.0459 \\
$\operatorname{imf}_{9}$ & 20.3512 \\
$\operatorname{imf}_{10}$ & 51.2981 \\
$\operatorname{imf}_{11}$ & 4.3109 \\
$\operatorname{imf}_{12}$ & 3.5685 \\
$\operatorname{imf}_{13}$ & 2.4170 \\
$\operatorname{imf}_{14}$ & 1.1268 \\
$\operatorname{imf}_{15}$ & 0.8334 \\
$\operatorname{imf}_{16}$ & 0.1514 \\
\hline
\end{tabular}

(2) The special "steel plate-buffer interlayer-steel plate" structure can effectively attenuate the explosion vibration effect. The main vibration frequency and vibration amplitude

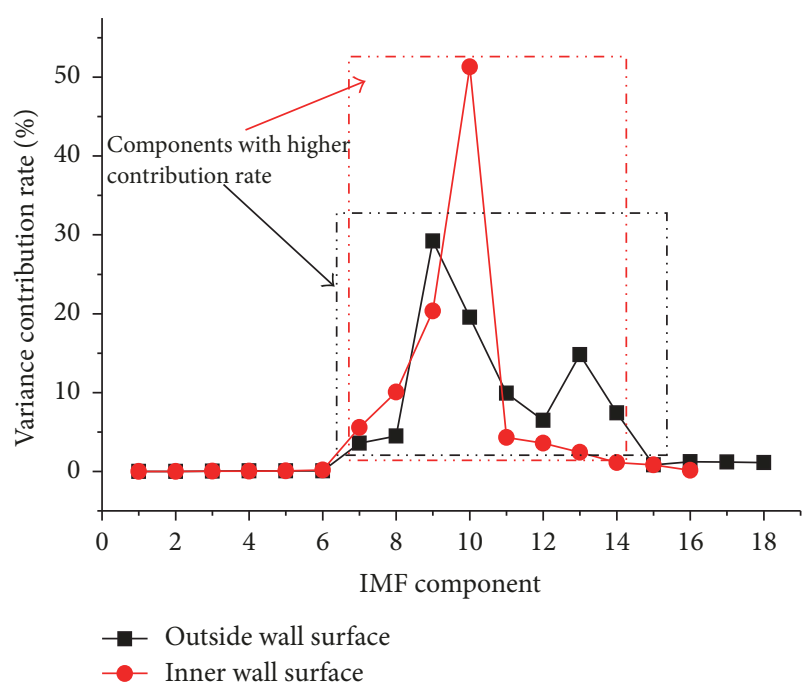

FIGURE 9: IMF components' variance contribution rates of vibration velocity signals at inner and outer surfaces of explosive vessel shell.

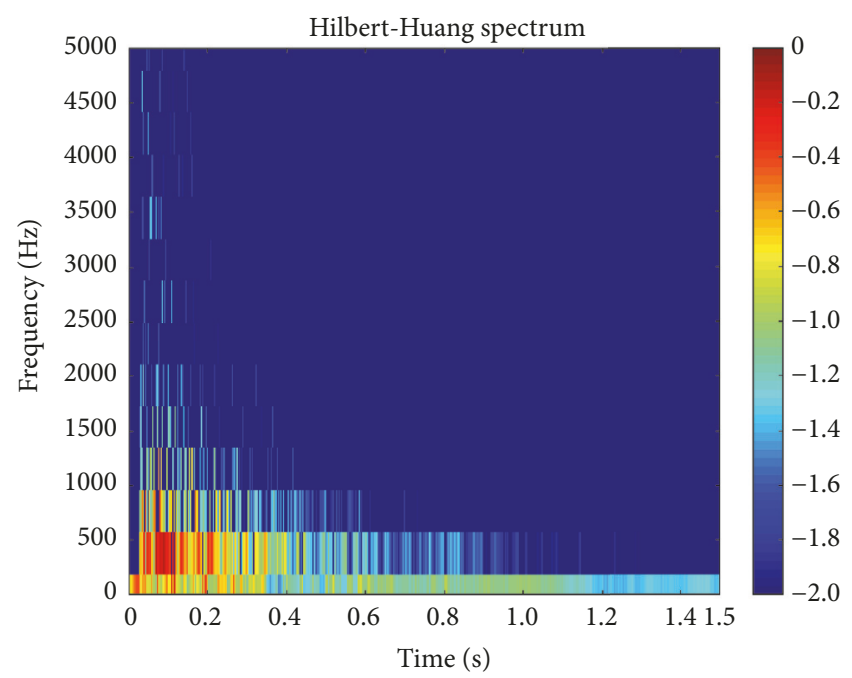

FIGURE 10: Frequency spectrum of vibration velocity signal at inner surface. Note. The color bar on the right represents the magnitude of vibration amplitude; the deeper the color, the greater the amplitude.

were apparently reduced, and the vibration acting time of low-order frequency was shortened.

(3) According to the spectrum analysis of vibration signals of explosive vessel, combining with the natural vibration frequency of the corresponding part, the vulnerable part in the process of application can be better analyzed, which provided a valuable reference for vibration reduction design and safer protection of explosive vessel.

\section{Conflicts of Interest}

The authors declare that they have no conflicts of interest. 


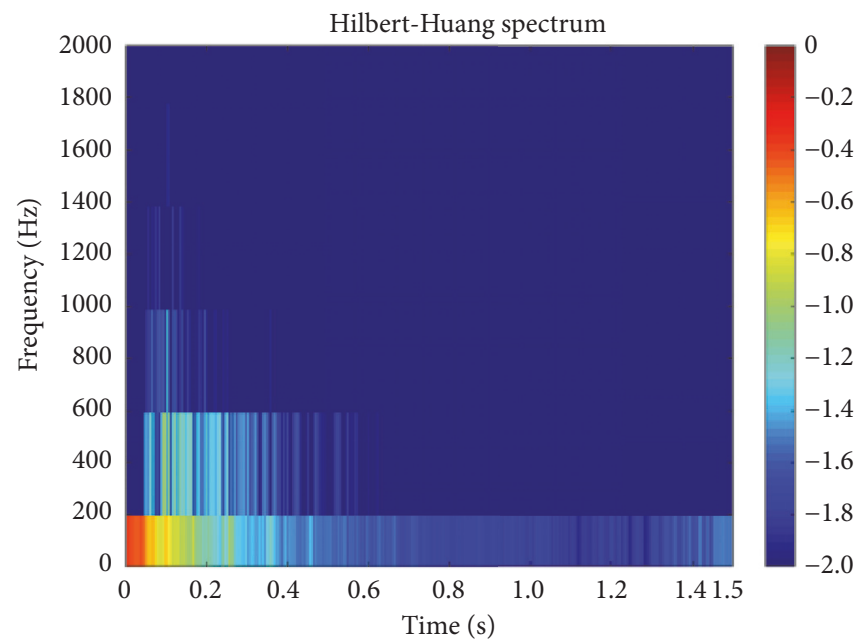

FIGURE 11: Frequency spectrum of vibration velocity signal at outer surface.

\section{Acknowledgments}

The authors gratefully acknowledge the financial support from the National Natural Science Foundation of China (no. 11502001), China Postdoctoral Science Foundation Funded Project (no. 2014M561808), and Postdoctoral Science Foundation Funded Project in Anhui Province (no. 2014B035).

\section{References}

[1] S. Y. Chen, B. Y. Hu, Y. Gu, and Q. Dong, "Experimental investigation on dynamic response of spherical explosive chamber," Acta Armamentarji, vol. 31, no. 4, pp. 504-509, 2010.

[2] J.-Y. Zheng, G.-D. Deng, Y.-J. Chen et al., "Experimental investigation on dynamic response and fracture characteristics of discrete multilayered thick-walled explosion containment vessels," Explosion and Shock Waves, vol. 25, no. 6, pp. 506-511, 2005.

[3] W. E. Baker, "The elastic-plastic response of thin spherical shells to internal blast loading," Journal of Applied Mechanics, vol. 27, no. 1, pp. 139-144, 1960.

[4] W. E. Baker, W. C. L. Hu, and T. R. Jackson, "Elastic response of thin spherical shells to axisymmetric blast loading," Journal of Applied Mechanics, vol. 33, no. 4, pp. 800-806, 1964.

[5] T. A. Duffey and C. Romero, "Strain growth in spherical explosive chambers subjected to internal blast loading," International Journal of Impact Engineering, vol. 28, no. 9, pp. 967-983, 2003.

[6] Y.-Y. Ma, J.-Y. Zheng, Y.-J. Chen, and G.-D. Deng, "Numerical simulation on dynamic response of the cylindrical explosion containment vessel with an elliptical cover," Explosion and Shock Waves, vol. 29, no. 3, pp. 249-254, 2009.

[7] Y.-J. Zhang, M.-P. Zhang, S.-L. Xu, and P. Zhang, "Numerical investigation on blast wave propagation and dynamic response of an explosion vessel," Explosion and Shock Waves, vol. 23, no. 4, pp. 331-336, 2003.

[8] N. Kambouchev, R. Radovitzky, and L. Noels, "Fluid-structure interaction effects in the dynamic response of free-standing plates to uniform shock loading," Journal of Applied Mechanics, vol. 74, no. 5, pp. 1042-1045, 2007.
[9] N. Kambouchev, L. Noels, and R. Radovitzky, "Numerical simulation of the fluid-structure interaction between air blast waves and free-standing plates," Computers \& Structures, vol. 85, no. 11-14, pp. 923-931, 2007.

[10] A. Vaziri and J. W. Hutchinson, "Metal sandwich plates subject to intense air shocks," International Journal of Solids and Structures, vol. 44, no. 6, pp. 2021-2035, 2007.

[11] Q. Dong, Q. M. Li, and J. Y. Zheng, "Further study on strain growth in spherical containment vessels subjected to internal blast loading," International Journal of Impact Engineering, vol. 37, no. 2, pp. 196-206, 2010.

[12] Q. M. Li, Q. Dong, and J. Y. Zheng, "Strain growth of the in-plane response in an elastic cylindrical shell," International Journal of Impact Engineering, vol. 35, no. 10, pp. 1130-1153, 2008.

[13] D. C. Lin, H. J. Shi, C. H. Bai, and Q. Zhan, "Time-frequency analysis of explosion seismic effects," Explosion and Shock Waves, vol. 23, no. 1, pp. 31-36, 2003.

[14] X. Qiu, X. Shi, J. Zhou, D. Huang, and X. Chen, "On vibration reduction effect of short millisecond blasting by high-precision detonator based on HHT energy spectrum," Explosion and Shock Waves, vol. 37, no. 1, pp. 107-113, 2017.

[15] T. L. Huang, M. L. Lou, H. P. Chen, and N. B. Wang, "An orthogonal Hilbert-Huang transform and its application in the spectral representation of earthquake accelerograms," Soil Dynamics and Earthquake Engineering, vol. 104, pp. 378-389, 2018.

[16] Z. Wang, C. Fang, Y. Chen, and W. Cheng, "A comparative study of delay time identification by vibration energy analysis in millisecond blasting," International Journal of Rock Mechanics and Mining Sciences, vol. 60, no. 8, pp. 389-400, 2013.

[17] A. Y. Ayenu-Prah and N. O. Attoh-Okine, "Comparative study of hilbert-huang transform, fourier transform and wavelet transform in pavement profile analysis," Vehicle System Dynamics, vol. 47, no. 4, pp. 437-456, 2009.

[18] G. Y. Xu, G. S. Zhong, and Z. M. Xiong, "Study and application of energy analysis method for blasting seismic safety based on wavelet transform," Chinese Journal of Geotechnical Engineering, vol. 28, no. 1, pp. 24-28, 2006.

[19] Z. K. Peng, P. W. Tse, and F. L. Chu, "A comparison study of improved Hilbert-Huang transform and wavelet transform: 
application to fault diagnosis for rolling bearing," Mechanical Systems and Signal Processing, vol. 19, no. 5, pp. 974-988, 2005.

[20] C. Q. Zhang, Y. F. Jia, and J. Kang, "Explosive blast test and distribution analysis based on HHT," Journal of Test and Measurement Technology, vol. 29, no. 6, pp. 473-478, 2015.

[21] N. E. Huang, "The empirical mode decomposition and Hilbert spectrum for nonlinear and non-stationary time series analysis," Proceedings of the Royal Society A, vol. 454, no. 1971, pp. 903-995, 1998.

[22] G. Kerschen, A. F. Vakakis, Y. S. Lee, D. M. McFarland, and L. A. Bergman, "Toward a fundamental understanding of the Hilbert-HUAng transform in nonlinear structural dynamics," Journal of Vibration and Control, vol. 14, no. 1-2, pp. 77-105, 2008.

[23] R. R. Zhang, S. Ma, E. Safak, and S. Hartzell, "HilbertHuang transform analysis of dynamic and earthquake motion recordings," Journal of Engineering Mechanics, vol. 129, no. 8, pp. 861-875, 2003.

[24] S. K. Lee, Y. W. Kim, M. H. Koo, H. I. Gimm, and H. H. Yoo, "Hilbert-Huang Transform (HHT) transient analysis of composite panel undergoing high-velocity impact," Journal of Mechanical Science and Technology, vol. 24, no. 12, pp. 23952400, 2010.

[25] W. Zhen-Xiong, G. Wen-Bin, L. Ting, L. Jian-Qing, X. JingLin, and L. Xin, "Blasting vibration generated by breakingblasting large barriers with EBBLB," Shock and Vibration, vol. 2016, Article ID 7503872, 13 pages, 2016.

[26] K. Kojima, K. Sakaguchi, and I. Takewaki, "Mechanism and bounding of earthquake energy input to building structure on surface ground subjected to engineering bedrock motion," Soil Dynamics and Earthquake Engineering, vol. 70, pp. 93-103, 2015.

[27] S. J. Loutridis, "Damage detection in gear systems using empirical mode decomposition," Engineering Structures, vol. 26, no. 12, pp. 1833-1841, 2004.

[28] X. B. Li, Y. P. Zhang, Z. X. Liu, Y. J. Zuo, W. H. Wang, and W. J. $\mathrm{Li}$, "Wavelet analysis and Hilbert-Huang transform of blasting vibration signal," Explosion and Shock Waves, vol. 25, no. 6, pp. 528-535, 2005.

[29] Y.-X. Zeng, C.-H. Bai, and Z.-Q. Wang, "Vibrating response of a residential structure to blast based on HHT," Journal of Vibration and Shock, vol. 33, no. 15, pp. 71-75, 2014.

[30] D. X. Wang, Y. L. Hu, J. Cao, and W. J. Li, "Research on the vibration characteristics of the cylindrical explosion vessel," Pressure Vessel Technology, vol. 24, no. 11, pp. 6-8, 2007.

[31] S. C. Stiros, "Errors in velocities and displacements deduced from accelerographs: an approach based on the theory of error propagation," Soil Dynamics and Earthquake Engineering, vol. 28, no. 5, pp. 415-420, 2008.

[32] K.-T. Park, S.-H. Kim, H.-S. Park, and K.-W. Lee, “The determination of bridge displacement using measured acceleration," Engineering Structures, vol. 27, no. 3, pp. 371-378, 2005.

[33] C.-H. Bai, Y.-X. Zeng, Z.-Q. Wang, and J. Chen, "HilbertHuang transform analyzing on the response of seismic waves to the explosive performance," Transactions of Beijing Institute of Technology, vol. 34, no. 12, pp. 1234-1239, 2014. 


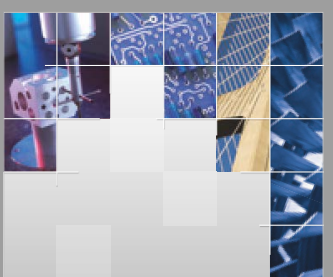

\section{Enfincering}
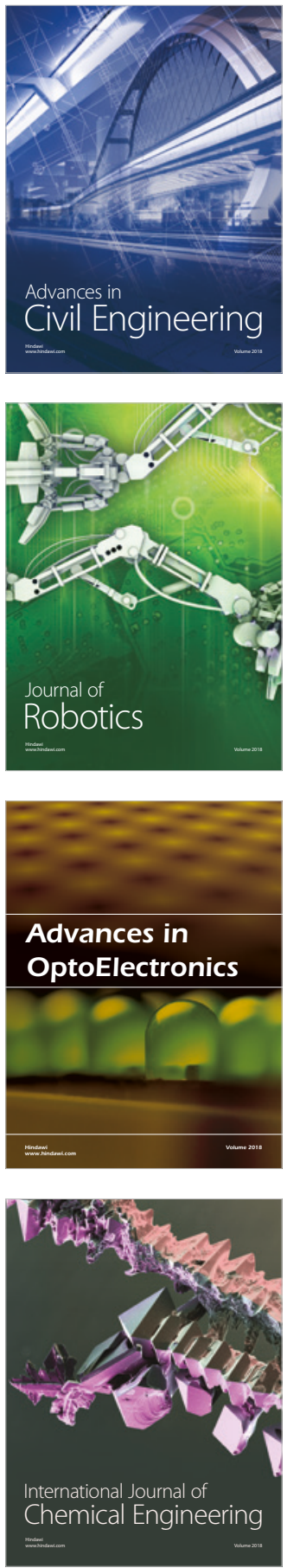

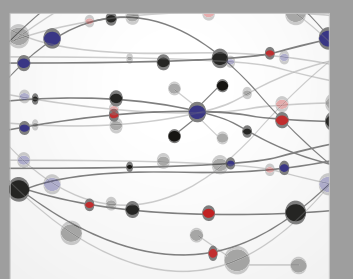

\section{Rotating \\ Machinery}

The Scientific World Journal

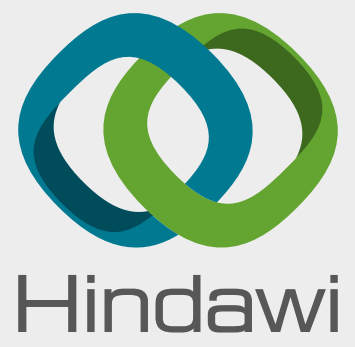

Submit your manuscripts at

www.hindawi.com
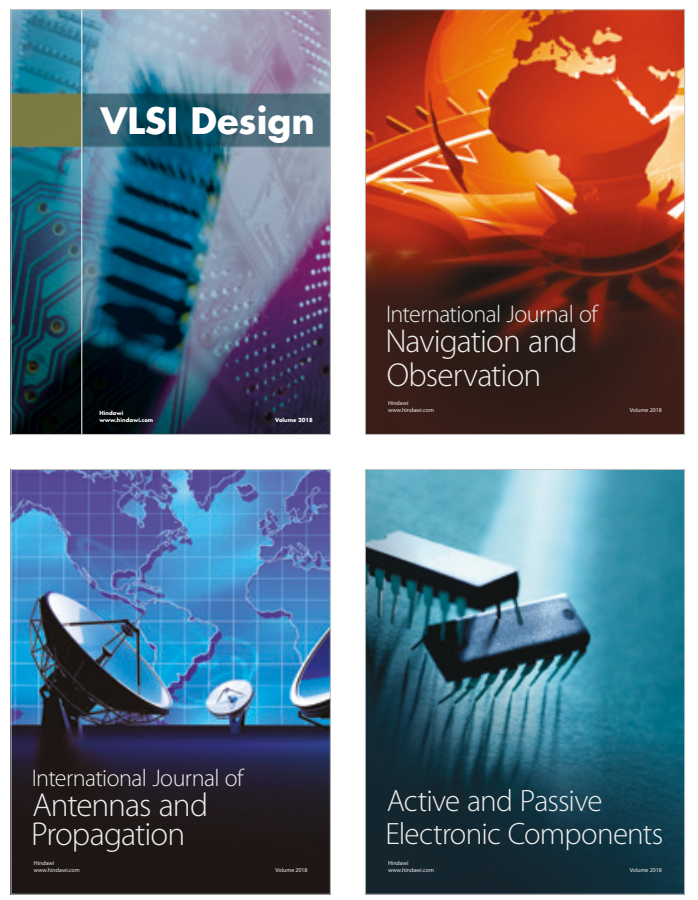
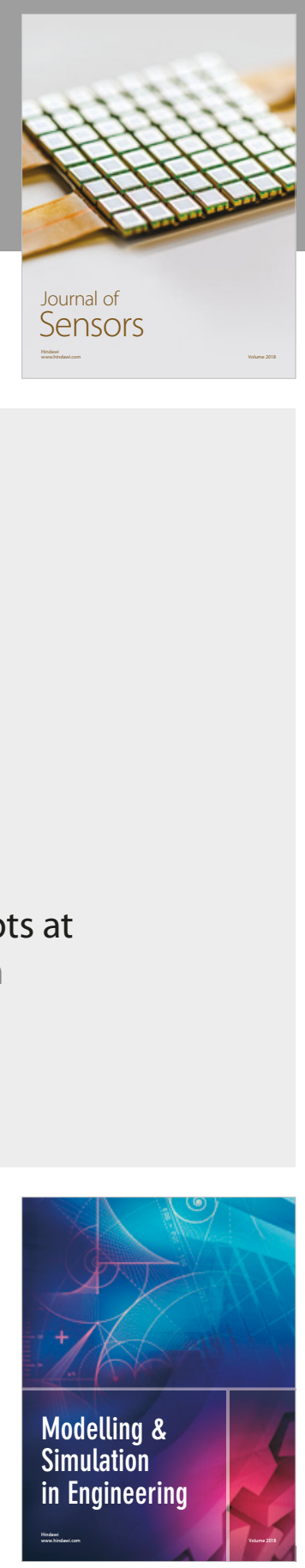

\section{Advances \\ Multimedia}
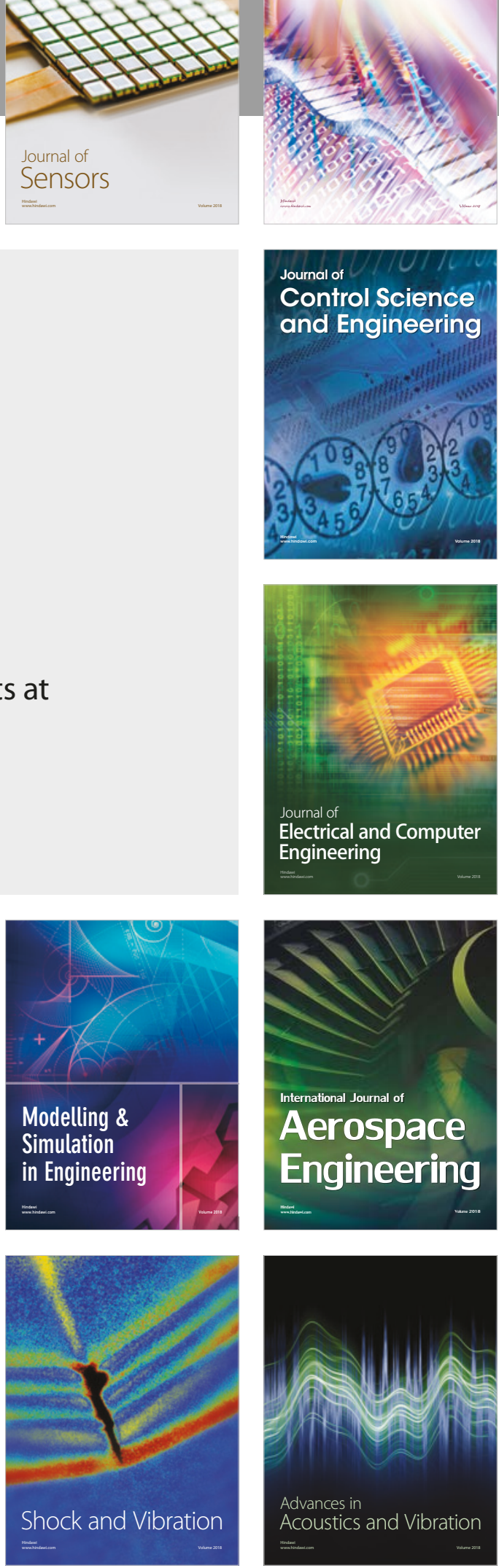